\title{
The BABAR LST Detector High Voltage System: Design and Implementation
}

\author{
Gabriele Benelli, Klaus Honscheid, Member, IEEE, Elizabeth A. Lewis, Joseph J. Regensburger, \\ and Dale S. Smith, Member, IEEE
}

\begin{abstract}
In 2004, the first two sextants of the new Limited Streamer Tube (LST) detector were installed in the BABAR experiment to replace the ageing Resistive Plate Chambers (RPCs) as active detectors for the BABAR Instrumented Flux Return (IFR) muon system. Each streamer tube of the new detector consists of 8 cells. The cell walls are coated with graphite paint and a $100 \mu \mathrm{m}$ wire forms the anode. These wires are coupled in pairs inside the tubes resulting in 4 independent twocell segments per LST. High voltage (HV) is applied to the 4 segments through a custom connector that also provides the decoupling capacitor to pick up the detector signals from the anode wires. The BABAR LST detector is operated at $5.5 \mathrm{kV}$. The high voltage system for the LST detector was designed and built at The Ohio State University (OSU HVPS). Each of the 25 supplies built for BaBar provides 80 output channels with individual current monitoring and overcurrent protection. For each group of 20 channels the HV can be adjusted between 0 and $6 \mathrm{kV}$. A 4-fold fan-out is integrated in the power supplies to provide a total of 320 outputs. The power supplies are controlled through built-in CANbus and Ethernet (TCP/IP) interfaces.

In this presentation we will discuss the design and novel features of the OSU HVPS system and its integration into the BABAR EPICS detector control framework. Experience with the supplies operation during the LST extensive quality control program and their performance during the initial data taking period will be discussed.
\end{abstract}

\section{INTRODUCTION}

$\Psi_{\mathrm{rap}}$ IMITED Streamer Tubes were chosen to replace the

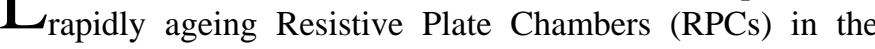
Instrumented Flux Return (IFR) muon system [1] of the $B A B A R$ detector [2]. The LST principle of operation is based on the ionization caused by a charged particle crossing the tube. The ionization causes a streamer discharge in the gas volume between the anode wire and the graphite coating of the cell and the induced signal on the HV wire can be measured. Since the High Voltage (HV) requirements of the new limited

Manuscript received November 11, 2005.

G. Benelli is with the Department of Physics, The Ohio State University, Columbus, OH 43210 USA (telephone: 650-926-2307, e-mail: gbenelli@slac.stanford.edu).

K. Honscheid is with the Department of Physics, The Ohio State University, Columbus, OH 43210 USA (telephone: 614-292-3287, e-mail: kh@mps.ohio-state.edu).

E. A. Lewis is with the Department of Physics, The Ohio State University, Columbus, OH 43210 USA (telephone: 614-292-9929, e-mail: elewis@mps.ohio-state.edu).

J. J. Regensburger is with the Department of Physics, The Ohio State University, Columbus, OH 43210 USA (telephone: 614-247-8296, e-mail: jregens@mps.ohio-state.edu).

D. S. Smith is with the Department of Physics, The Ohio State University, Columbus, OH 43210 USA (telephone: 614-292-9929, e-mail: ssmith@mps.ohio-state.edu). streamer tubes were incompatible with the previous detector, a new HV system had to be designed.

\section{High Voltage ReQuirements}

The BABAR LSTs are operated with a gas mixture of Argon, Isobutane, and Carbon Dioxide (3:8:89). The active $\mathrm{HV}$ region ranges between 5 and $6 \mathrm{kV}$, with a typical working point of $5.5 \mathrm{kV}$. At this operating voltage, currents in a single LST tube typically range between 15 and 100nA without beams, and between 50 and 1000nA (depending on the position within the detector) with colliding beams. Since the occupancy and hence the current will vary as function of the distance to the beam pipe it is a requirement that the HV operating point can be adjusted at least on a layer by layer basis.

The BABAR LST detector consists of 1164 tubes, each of them split further into 4 two-cell segments, for a total of 4656 independent HV detection elements, requiring an HV system with a very large number of outputs. The high level of granularity makes the LST detector, as a whole, less sensitive to a single cell failure if the high granularity is matched by the HV system and individual HV channels can be disconnected or treated separately.

The current drawn by an individual LST tube is a critical parameter to monitor the performance of the detector. Therefore, independent current monitors for each of the 1164 detector elements are required. Each current has to be measured with a precision better than $10 \mathrm{nA}$, at a rate of $10 \mathrm{~Hz}$ or more.

A sudden current increase to values higher than 2000nA is generally a sign of a self-sustaining discharge process in one of the tube cells. A hardware overcurrent protection, that stops the discharge by automatically lowering the high voltage, is necessary to protect the detector. For other high current situations such as high, beam-induced backgrounds, a sophisticated and configurable trip logic needs to be implemented.

While high granularity in terms of $\mathrm{HV}$ outputs and of current monitoring is necessary, independent $\mathrm{HV}$ operation of the individual two-cell segments within a tube is not needed. Furthermore, HV control granularity is reduced by the characteristics of the BABAR detector, where the tubes are radially distributed in 12 layers, containing up to 20 tubes each. Since the current drawn by a tube is proportional to the counting rates, i.e. the exposure to radiation and background, layers closer to the interaction point are expected to draw higher current than the outer ones. High currents are damaging in terms of the tubes lifetime, so the HV output must be 
adjustable, in order to allow minimization of the currents without compromising detection efficiency.

Finally, the LST HV system has to be fully integrated in the $B A B A R$ detector control system which is based on the EPICS framework.

\section{OSU High Voltage Power Supply}

The Ohio State University High Voltage power supply (OSU HVPS, Fig. 1) is a cost effective solution that fulfills all of the requirements listed in the previous section.

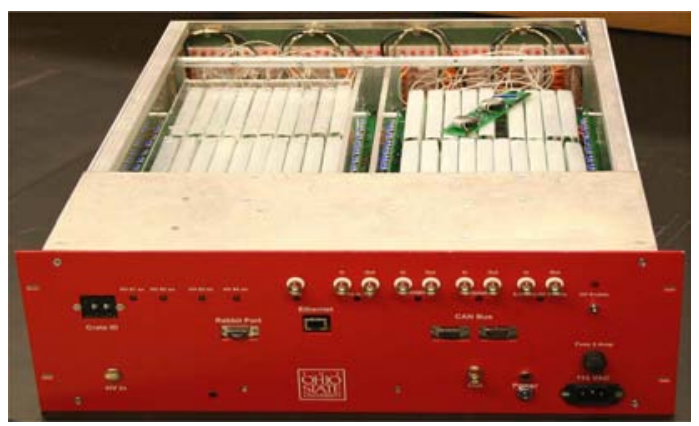

Fig. 1. OSU HVPS with top cover and RF shields removed. One current monitor module has been extracted.

It was designed to provide $320 \mathrm{HV}$ outputs, with variable output voltage between 0 and $6 \mathrm{kV}$. In terms of $\mathrm{HV}$ control the outputs are grouped into 4 independent $\mathrm{HV}$ groups of 80 outputs. Groups of 4 outputs are connected to one of 80 current monitor circuits in the power supply. This matches the design of the BABAR LST tubes where the 8 anode wires are combined internally to 4 readout/HV channels. As a result we have one current monitor per LST tube. Even though the 4 outputs are driven in parallel by the power supply, having 4 independent output connections preserves the ability to disconnect and treat separately the individual 2-wire segments of the LST tubes.

\section{A. Current Measurement}

At the hardware level the current monitoring is carried out by 80 current monitor modules (Fig. 2).

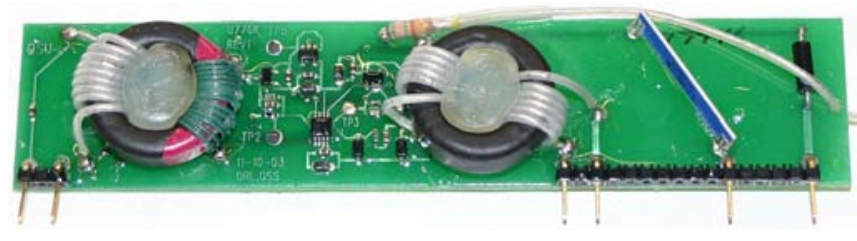

Fig. 2. OSU HVPS current monitoring module.

Each module is capable of measuring a full scale current of $12 \mu \mathrm{A}$ with a resolution of $1.5 \mathrm{nA}$, and it consists of a floating $+5 \mathrm{~V}$ (DC) supply, a low power ADC circuit, and output protection circuitry. The floating $+5 \mathrm{~V}$ (DC) supply is referenced to the module individual output voltage. This allows the current measurement circuitry to operate at all output voltage settings and protects the circuit during unexpected shorts on the output. The floating ADC circuit (Fig. 3) consists of a sense resistor in series with the output, an operational amplifier to measure and buffer the voltage drop across the sense resistor, and a voltage controlled oscillator (VCO) that generates a frequency proportional to the measured voltage from the operational amplifier.

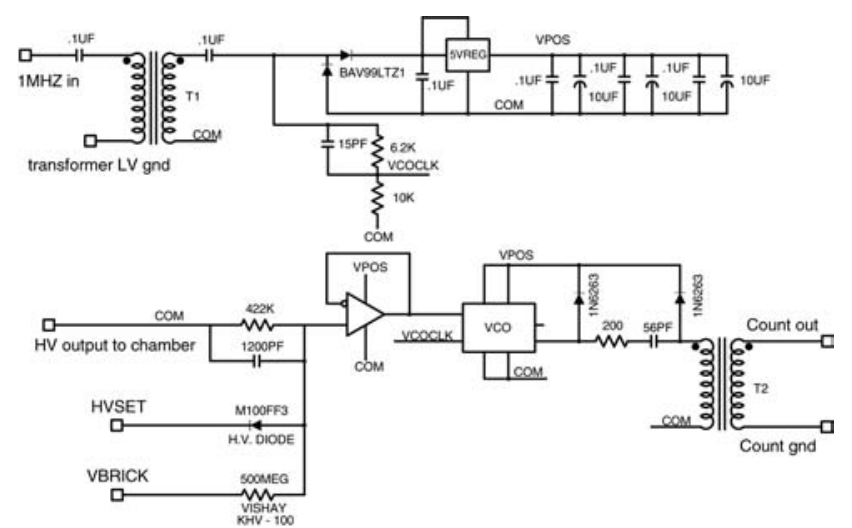

Fig. 3. OSU HVPS current monitoring module circuit diagram.

The signal from the VCO is referenced back to (low voltage) ground through an isolation transformer, where it is then amplified and its frequency counted by a Xilinx FPGA.

\section{B. Overcurrent protection}

An overcurrent protection circuit is part of each current monitoring module and is implemented using an $\mathrm{HV}$ diode as shown in Fig. 4.

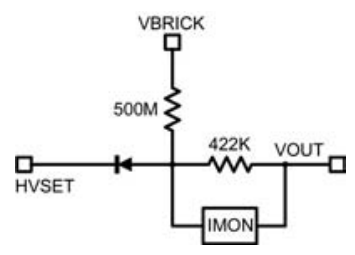

Fig. 4. Overcurrent protection circuit diagram

During normal operation, the output current is small and the HV diode is forward biased. The forward bias condition holds the output voltage equal to the set voltage plus the high voltage diode drop. When the output current rises above the overcurrent protection limit ( $I_{\text {Thresh }}$ ) determined by the DCDC converter voltage (VBRICK ), the $500 \mathrm{M} \Omega$ resistor, and the high voltage set point ( HVSET), the diode becomes reverse biased. The overcurrent protection limit is determined by:

$$
I_{\text {Thresh }}=(\text { VBRICK }- \text { HVSET }) / 500 M \Omega \text {. }
$$

If the output current demand continues to increase, the output voltage drops linearly with a slope determined by the $500 \mathrm{M} \Omega$ resistor.

This overcurrent protection mechanism is based on the LST operation principle to quickly and automatically recover tubes that go in self-sustaining discharge mode. The affected tube 
draws a current well above $I_{\text {Thresh }}$ and as a result its voltage is lowered linearly, until it drops below the active HV region. When the discharge stops, the tube is automatically ramped back to its operational HV. This mechanism provides each channel with overcurrent protection without affecting any other channel in the same supply.

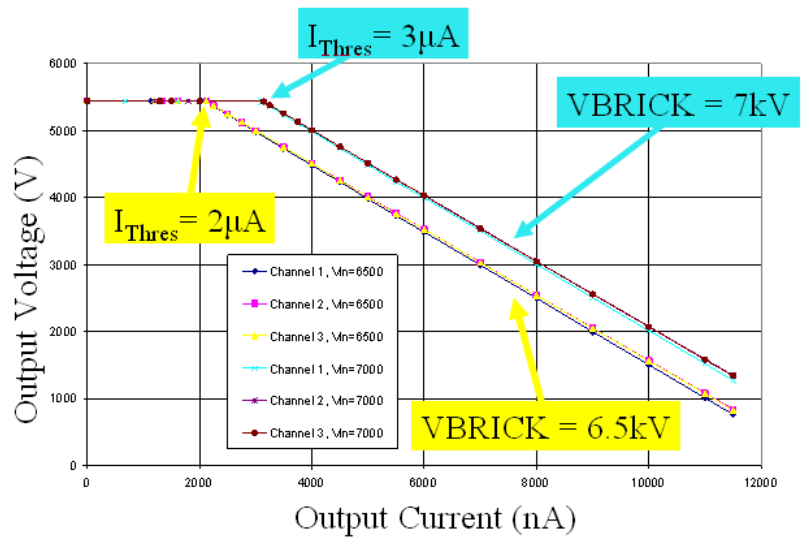

Fig. 5. Output voltage vs. output current for a HV channel of the OSU HVPS. The plot illustrates the overcurrent protection mechanism, and the overcurrent threshold ( $\mathrm{I}_{\text {Thresh }}$ ) dependence from the DC-DC converter output voltage (VBRICK).

Since the internal high voltage DC-DC converter can be set remotely, it is possible to vary the current protection limit $I_{\text {Thresh }}$ to adapt to in changing operating conditions, without hardware modifications (Fig. 5).

\section{Voltage Regulation}

The HV rail for each HVPS is supplied by an internal Ultravolt ${ }^{\circledR}$ DC-DC converter that can provide up to $10 \mathrm{kV}$ at $3 \mathrm{~mA}$ [3]. The individual $\mathrm{HV}$ group voltages are derived from the DC-DC converter and controlled by a Xilinx® ${ }^{\circledR}$ FPGA through 12-Bit DACs (Fig.6). The analog output voltage from each DAC is used as a reference for an operational amplifier. The operational amplifier uses a precision voltage divider network to obtain a measurement of the output voltage and sets the current in a high voltage BJT accordingly to match the output measurement to the reference setting. The current in the BJT flows through a string of FET's, used to safely divide the output voltage, and through the high voltage input resistor from the DC-DC converter. When the BJT current is set to a large value, the voltage drop across the input resistor makes the high voltage output go low. When little current is flowing in the BJT and FET string, the drop on the input resistor is small and the individual segment voltage is close to that of the DC-DC converter. To provide a measure of the individual output voltages, an ADC circuit similar to the one used on the current measurement modules is attached to the same precision voltage divider network mentioned above. The HVPS is capable of measuring the individual output voltages with $1 \mathrm{~V}$ resolution.

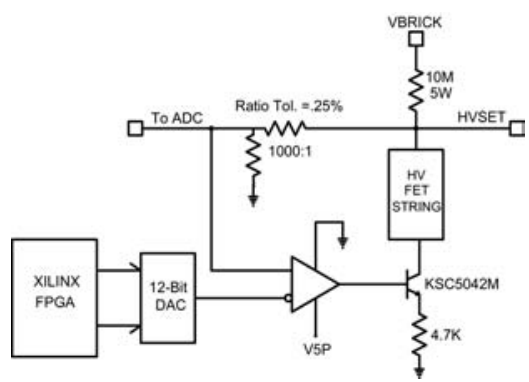

Fig. 6. Voltage regulation circuit diagram.

\section{Back-Panel Fan-Out}

It was already mentioned that the $B A B A R$ LST tubes consist of 8 individual cells and that pairs of cells are combined to form 4 readout/HV channels. In order to match this design a 1-to-4 fan-out (Fig. 7) is integrated into backpanel of the HV power supply system.

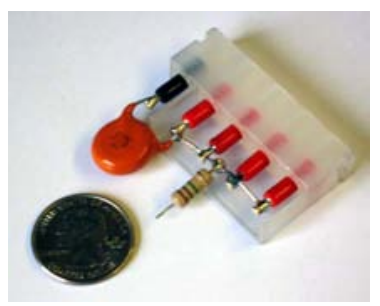

Fig.7 The OSU HVPS custom designed connector with four 2-mm Banana plugs (HV) and one ground connections embedded in a injection-molded polyethylene piece.

\section{E. Digital board and embedded software}

A Xilinx ${ }^{\circledR}$ FPGA performs the data collection and the control signal generation. A Rabbit Semiconductor ${ }^{\circledR}$ microcontroller, RCM 3200 [4], is interfaced to the Xilinx ${ }^{\circledR}$ chip to control the power supply and to implement higher level logic and communication functions. Calibration information and settings are kept in the microcontroller flash memory. The microcontroller processes the current and voltage information provided by the FPGA in order to implement the ramping and trip logic. The command structure is implemented at the microcontroller level using the two communication interfaces available, Ethernet (TCP/IP) and CANbus. The software was fully developed at The Ohio State University, and the ability to modify and continue development to adapt to experimental necessities is a definite advantage over a commercial solution.

\section{F. Trip and Ramping logic}

In order to protect the LST system from high current situations that could damage the detector, the OSU HVPS include a sophisticated, configurable trip logic covering three possible trip mechanisms: current over threshold for a user selectable amount of time, instantaneous current over threshold (spike), and an over-current situation for the internal Ultravolt ${ }^{\circledR}$ DC-DC converter. Both the time over threshold and spike trip are implemented for each individual output channel. For the time over threshold trip logic, trip levels and 
time over threshold limits can be differentiated for ramping and non-ramping conditions.

\section{Detector CONTROLS}

The OSU HVPS controls are fully integrated in the BABAR EPICS detector control system using the CANbus interface. A dedicated Motorola ${ }^{\circledR}$ MVME5500 [5] Input Output Controller (IOC) running RTEMS ${ }^{\circledR}$ [6] is used to run the LST HV EPICS application. The EPICS controls allow prompt reaction to changing beam conditions and safe operation of the LST detector during injection cycles. The detector controls include an integrated alarm system with configurable warning and alarm levels for all quantities monitored by the OSU HVPS. An archiving function is used to record the monitored quantities in a database. A Graphical User Interface is available to operate the OSU HVPS in stand alone mode.

A series of opto-coupled input/output signals are provided on the front panel of the OSU HVPS to integrate the LST HV system in the BaBar hardware interlock system that prevents the accelerator from injecting beam when the detector conditions are not safe.

A redundant interlock system is implemented that inhibits turning on high voltage when conditions are not safe: an external HV enable signal controlled by $B A B A R$, a front panel HV enable switch, a software HV enable, a switch lock power switch with a removable key. For the supply to provide HV output, all interlocks must be set accordingly.

\section{RUNNING EXPERIENCE}

A total of 25 OSU HV power supplies have been constructed: 23 are located at the experiment location, the Stanford Linear Accelerator Center (SLAC), 2 are kept at OSU. The use of the OSU HVPS allowed for a very successful LST Quality Control (QC) program. HV was a key component of the QC program and it was used for HV conditioning, burnin tests, taking single rate plateaus curves and to run a scan test with a radioactive source.

Data taking with the new LST detector started in April 2005 and the new power supplies have performed successfully. So far, only the top and bottom sextants of the LST detector have been installed (the remaining sextants will be installed in 2006). The LSTs in each sextant are powered by 3 OSU HVPS. An extra OSU HVPS, the so called hospital, is used to study and treat any misbehaving tubes from the two sextants.

Overall, the LST detector has performed very well. Only a few tubes showed signs of internal discharges and additional $\mathrm{HV}$ conditioning using the hospital supply recovered most of them. Very few channels $(<0.3 \%)$ had to be permanently operated at a lower voltage.

The innermost layers drew very high currents as a function of luminosity (Fig. 8), so the first two layers tubes were split into two OSU HVPS channels. Extrapolating from Fig. 8, the current drawn per tube at a luminosity of $2 \times 10^{34} \mathrm{~cm}^{-2} \mathrm{~s}^{-1}$, which would be the highest luminosity $B A B A R$ is expected to run at, is approximately $8000 \mathrm{nA}$. This is a factor of 4 larger than originally expected, but with a firmware upgrade the OSU HV supplies will be able to power the LSTs even in this scenario.

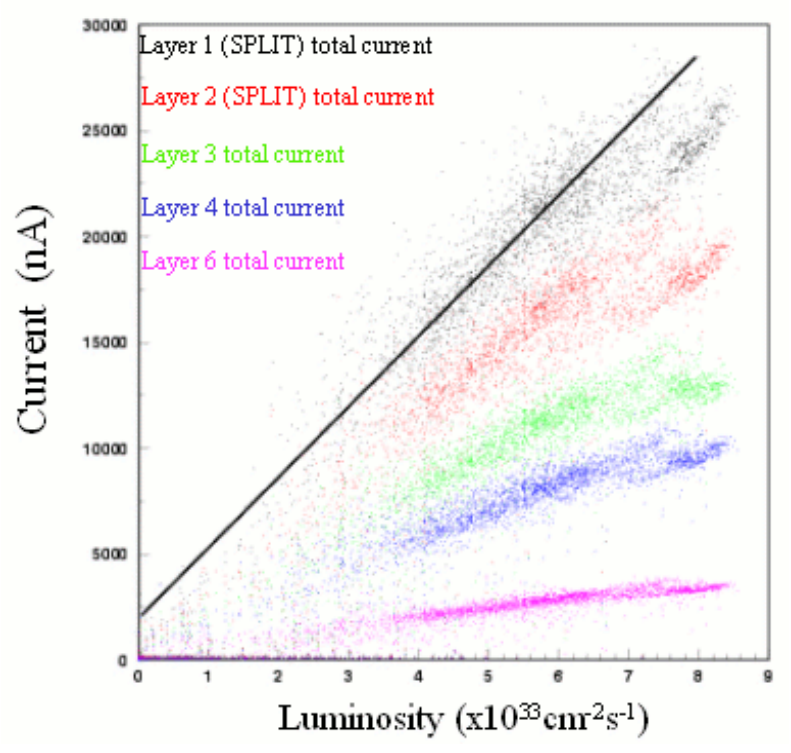

Fig. 8. Total layer current vs. luminosity. The plot shows how the current drawn by tubes is linearly dependent on luminosity. The slope for the innermost layers is much steeper than the middle layers. Extrapolating the current drawn per tube at $2 \times 10^{34} \mathrm{~cm}^{-2} \mathrm{~s}^{-1}$ would be approximately $8000 \mathrm{nA}$ per tube (a factor of 4 the original estimate).

\section{CONCLUSIONS}

A new, multi-channel power supply system was developed for the BABAR LST detector. The OSU HVPS system has proven to be a versatile and robust HV solution at much lower costs than other commercially available systems.

Performance during the QC and data taking is excellent, especially in terms of flexibility of the custom solution, which allows the system to adapt changing conditions, such as the higher than anticipated current running conditions.

\section{ACKNOWLEDGMENT}

The authors would like to thank the entire BABAR LST group and the members of the electronics workshop at The Ohio State University.

\section{REFERENCES}

[1] W. Menges for the BABAR-LST Collaboration, "The BABAR Muon System Upgrade," these proceedings.

[2] B. Aubert et al. [BABAR Collaboration], Nucl. Instrum. Meth. A 479, 1 (2002) [arXiv:hep-ex/0105044].

[3] Ultravolt, Inc (see http://www.ultravolt.com)

[4] Rabbit Semiconductor, Inc (see http://www.rabbitsemiconductor.com)

[5] Motorola, Inc (see http://www.motorola.com/content/0,,5655,00.html)

[6] RTEMS (see http://www.rtems.com/) 